\title{
A Novel Symmetrical Split Ring Resonator Based on Microstrip for Microwave Sensors
}

\author{
Rammah A. Alahnomi ${ }^{1}$, Z. Zakaria ${ }^{1}$, E. Ruslan ${ }^{2}$, Amyrul Azuan Mohd Bahar ${ }^{1}$ \\ ${ }^{1}$ Centre for Telecommunication Research and Innovation (CeTRI), Universiti TeKnikal Malaysia Melaka (UTeM), 76100 \\ Durian Tunggal, Melaka, Malaysia. Email: (alrammah89@gmail.com, zahriladha@utem.edu.my) \\ ${ }^{2}$ Faculty of Technology Engineering (FTK), Universiti TeKnikal Malaysia Melaka (UTeM), 76100 Durian Tunggal, Melaka, \\ Malaysia.
}

\begin{abstract}
In this paper, novel symmetrical split ring resonator (SSRR) is proposed as a suitable component for performance enhancement of microwave sensors. SSRR has been employed for enhancing the insertion loss of the microwave sensors. Using the same device area, we can achieve a high Q-factor of 141.54 from the periphery enhancement using Quasi-linear coupling SSRR, whereas loose coupling SSRR can achieve a Q-factor of 33.98 only. Using Quasi-linear coupling SSRR, the Q-factor is enhanced 4.16 times the loose coupling SSRR using the same device area. After the optimization was made, the SSRR sensor with loose coupling scheme has achieved a very high Qfactor value around 407.34 while quasi-linear scheme has achieved high Q-factor value of 278.78 at the same operating frequency with smaller insertion loss. Spurious passbands at 1st, 2nd, 3rd, and 4th harmonics have been completely suppressed well above $-20 \mathrm{~dB}$ rejection level without visible changes in the passband filter characteristics. The most significant of using SSRR is to be used for various industrial applications such as food industry, quality control, bio-sensing medicine and pharmacy. The simulation result that Quasi-linear coupling SSRR is a viable candidate for the performance enhancement of microwave sensors has been verified.
\end{abstract}

Keywords: Material characterization, microwave sensor, Symmetrical split ring resonator.

\section{INTRODUCTION}

Recently, significant advances have been done for material characterization using properties techniques. Some of these techniques are presented for the real part of the dielectric constant measurement; conversely, each technique is suitable and valuable for certain kinds of frequency ranges and certain kinds of materials. Material characterization has an application in various areas such as bio-sensing, quality control in the food industry, substrate properties and so on. Many methods have been proposed and used material characterization. These methods can be categorized as nearfiled sensors, transmission line, free-space methods, and resonant cavity [1]. Even though the microwave sensors are deployed for dielectric measurements for materials characterization, they are also used for measuring chemical reactions, humidity, and paramagnetic impurities. The main advantages of the microwave resonators used as sensors are: high precision level, high Q-factor, easy to design and fabricate, safe to use, reliable, low-cost, high measurement performance and sensitivity, and operating at various distinguished microwave frequencies [2]-[4]. Due to the permittivity that depends on the volumetric moisture content in any dielectric low loss material, the microstrip architecture is suitable for the sensor realization and this happens when the propagation of the electromagnetic wave along a microstrip depends on the medium that surrounds it.

Meat quality assessment by using dielectric properties has gained more research interest in the past few years. Checking the freshness and quality of the meat has various advantages which are effective, easy, rapid, non-destructive and reliable [5]. Various research studies have been focused on meat properties for lower frequencies. Meat quality aspects, quality classes, detection of frozen, chemical contamination and microbial activities have been characterized using the impedance variations of Kilo-Hertz to Mega-Hertz frequency ranges. However, moderately a few literature sources are found in Giga-Hertz ranges, which focus mainly on the assessment of freshness, salting process, aging state and fat determination [5]-[16].

In this paper, we will present the high Q-factor of a resonator based on symmetrical split ring resonator (SSRR). The SSRR has a very narrow bandwidth of the reflected signals which increase the Q-factor. These new SSRR are then used to suppress the spurious passbands of the stepped impedance low pass filters and they can be used for harmonic suppression of other types of filters as well. For further enhancement of the insertion and return loss, 
periphery is also incorporated between the two coupling gaps. The main contributions of this paper are to propose a symmetrical split ring resonator (SSRR) as a possible candidate for enhancing microwave sensor performance, design, fabrication and testing of harmonic suppressed filters using SSRR.

\section{PARAMETRIC STUDY OF SSRR SENSOR}

The geometry of the proposed SSRR structure is depicted in Fig.1.a) and Fig.1.b). Fig.1.a) is without overlay sample and Fig.1.b) is with an overlay sample view of the SSRR printed on the ground plane exactly beneath the $50 \Omega$ microstrip line. It consists of a symmetrical split ring resonator unlike SRR structure, which has slits ring resonators. The SSRR circuit dimensions are $100 \mathrm{~mm} \mathrm{x}$ $68 \mathrm{~mm} \times 0.787 \mathrm{~mm}(\operatorname{Lg} \times \mathrm{Wg} \times \mathrm{h})$, respectively, with the rings resonator radius $\mathrm{Ro}=15.85 \mathrm{~mm}$ and $\mathrm{Ri}=10.58 \mathrm{~mm}$ and line length $\mathrm{L}=34 \mathrm{~mm}$ patched on the substrate plane of a microstrip SSRR circuit printed on Roger 5880 dielectric substrate of $\varepsilon_{\mathrm{r}}=2.2$ and height of $0.787 \mathrm{~mm}$ [4], the resonance frequency is observed as $2.2 \mathrm{GHz}$ from HFSS High Frequency Structure Simulator (indicated in Fig.3.). It has been shown that the mean circumference of the ring resonator is equal to an integral of the guided wavelength as indicated below:

$$
2 \pi r=n \lambda g
$$

Where $n=1,2,3 \ldots$, and

$$
\lambda g=\frac{\lambda}{\sqrt{\varepsilon_{\text {eff }}}}
$$

So, the close-form formula for the resonance frequency of SSRR is given by:

$$
f_{o}=\frac{n c}{2 \pi r \sqrt{\varepsilon_{\text {eff }}}}
$$

Where c is the speed of light in vacuum and $\varepsilon_{\text {eff }}$ is the effective of dielectric constant.

It can be considered to be a simple LC circuit with a response frequency fr, using the following equation [17], [18]:

$$
f_{o}=\frac{1}{2 \pi \sqrt{L_{r}\left(\mathrm{C}_{s}+\mathrm{C}_{r}\right)}}
$$

Where, fr is the SSRR resonant frequency, Lr and Cr are the inductance and capacitance associated with SSRR, respectively, Cs is the capacitance between the ground plane and microstrip-line. The Cs and Lr are always considered as constant due to the material under test (MUT) which is usually non-magnetic and a constant permittivity is used in the substrate of the proposed sensor.
The quality factor (Q-factor) can be determined using this formula [17]:

$$
Q=\frac{\omega_{o}}{\Delta \omega}
$$

Where: $\omega$ is the center frequency and $\Delta \omega$ is the bandwidth. Note that SSRR is resonating at half of the guidedwavelength and dimension of the SSRR we have calculated for the resonance frequency in (2) which is demonstrated in Fig.1.a). For the sample, it is located at the maximum electric field which is indicated in Fig.1.b).

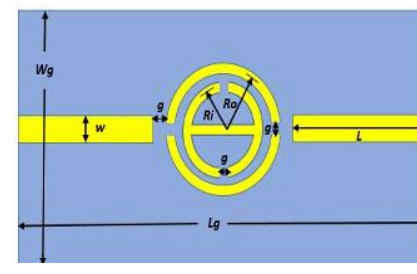

a)

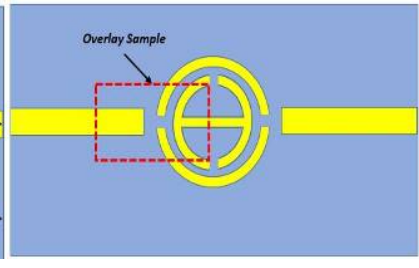

b)
Fig.1. Geometry of SSRR design (a) without an overlay meat sample (b) with an overlay meat sample.

Moreover, there is a modification to increase the coupling strength and efficiency. These coupling schemes can be found in literature [4], [5]. Scheme in Fig.2.a) is known as loose coupling. In this method, since the coupling strength is not much higher, its effect on resonance frequency is minimal. But the insertion loss is much higher, however, by selecting optimum gap size it can be reduced [19]. To increase the coupling strength and energy of the structure, a Quasi-linear coupling is used as illustrated in Fig.2.b). This method increases the coupling efficiency by embedding feed lines into the ring structure. It reduces the insertion loss unlike the loose coupling scheme.

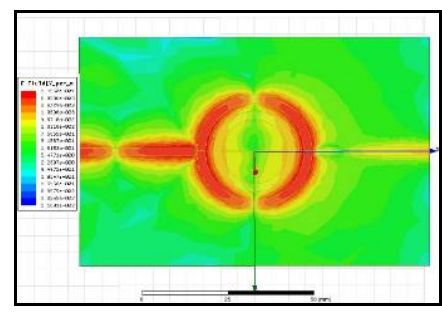

a)

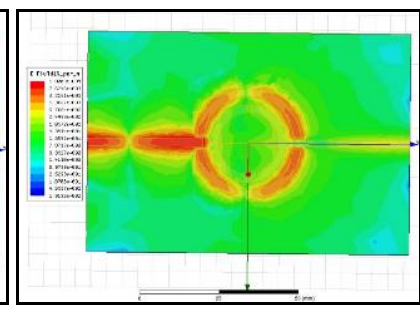

b)
Fig.2. Microwave biosensor of SSRR resonator: a) Loose coupling scheme, b) Quasi-linear scheme.

\section{RESUlTS AND DisCUSSION}

\section{A. Result of unloaded overlay sample}

Simulation result of symmetrical split ring resonator without overlay meat sample for the scattering parameters (S11 and S21 in $\mathrm{dB}$ ) is demonstrated in Fig.3.a) and Fig.3.b). As expected, without an overlay meat sample the resonator illustrates a periodic resonance at $2.2 \mathrm{GHz}$ 
intervals with unloaded condition resonator. During the simulation, the first resonance occurred at $2.26 \mathrm{GHz}$ for given range, up to $10 \mathrm{GHz}$, whereas, the second periodic resonance occurred at $4.4 \mathrm{GHz}$ and then the third at $6.6 \mathrm{GHz}$ and the last resonance occurred at $8.76 \mathrm{GHz}$. While at these periodic resonances, the result of S21 seems lossier when using coupling gap in SSRR. This is due to the loose coupling of the resonator. However, the Quasi-Linear coupling is used to overcome this problem. Whereas, the insertion loss is much better as it can be seen in Fig.3.a), it demonstrates the comparison of insertion loss S21 (dB) between the loose coupling and Quasi-linear coupling for the simulation results, where loose coupling has $-40 \mathrm{~dB}$ at 2.26 GHz while Quasi-linear coupling has a $-10 \mathrm{~dB}$ only. This indicates that a Quasi-Linear coupling has smaller insertion loss with high Q-factor compared to the loose coupling. Fig.3.b) demonstrates the return loss of the designed microwave resonator for both schemes, loose and Quasi-linear coupling. The results show that the Quasilinear has much better performance compared to loose coupling result. This is due to the gap coupling between the feed lines and the ring, whereas the larger gap will produce a minimum field perturbation with less sensitivity.

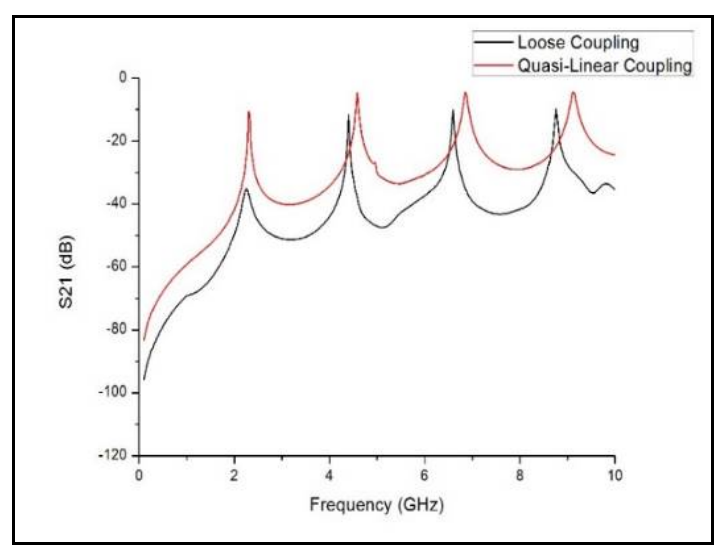

a)

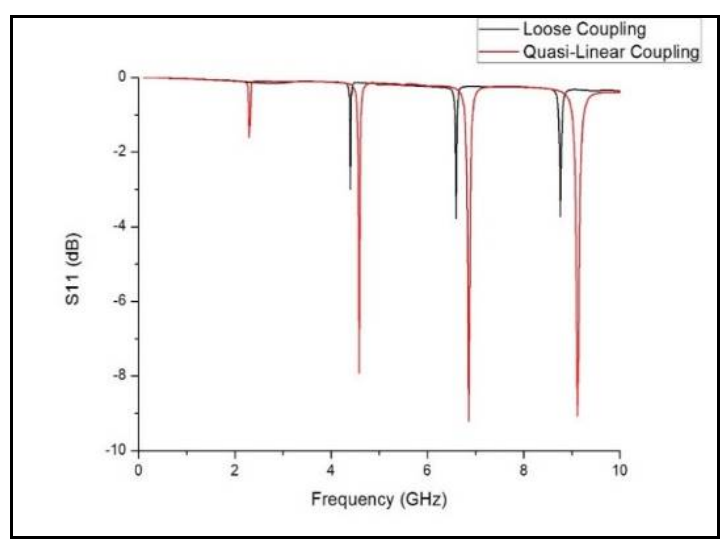

b)

Fig.3. Measured scattering parameters of unloaded resonator: a) insertion loss S21 (dB), b) return loss S11 (dB).
Table 1. Comparison between loose and Quasi-linear coupling in term of Q-factor, S11, and S21.

\begin{tabular}{|c|c|c|c|c|}
\hline \multirow{2}{*}{$\begin{array}{c}\text { Mode } \\
\text { (n) }\end{array}$} & $\begin{array}{c}\text { Frequency } \\
\text { (GHz) }\end{array}$ & $\begin{array}{c}\text { Q- } \\
\text { Factor }\end{array}$ & $\begin{array}{c}\text { S11 } \\
\text { (dB) }\end{array}$ & $\begin{array}{c}\text { S21 } \\
\text { (dB) }\end{array}$ \\
\hline 1 & 2.26 & 33.98 & -0.12 & -35.19 \\
\hline 2 & 4.4 & 517.65 & -2.99 & -11.61 \\
\hline 3 & 6.6 & 379.31 & -3.78 & -10.09 \\
\hline 4 & 8.76 & 369.62 & -3.74 & -9.66 \\
\hline
\end{tabular}

Table 2. Comparison between loose and Quasi-linear coupling in term of Q-factor, S11, and S21.

\begin{tabular}{|c|c|c|c|c|}
\hline \multirow{2}{*}{$\begin{array}{c}\text { Mode } \\
\text { (n) }\end{array}$} & \multicolumn{4}{|c|}{ Quasi-Linear Coupling } \\
\cline { 2 - 5 } & $\begin{array}{c}\text { Frequency } \\
\text { (GHz) }\end{array}$ & Q-Factor & $\begin{array}{c}\text { S11 } \\
\text { (dB) }\end{array}$ & $\begin{array}{c}\text { S21 } \\
\text { (dB) }\end{array}$ \\
\hline 1 & 2.30 & 141.54 & -1.61 & -10.51 \\
\hline 2 & 4.58 & 225.06 & -7.92 & -4.61 \\
\hline 3 & 6.86 & 170.86 & -9.21 & -4.39 \\
\hline 4 & 9.12 & 162.71 & -9.07 & -4.44 \\
\hline
\end{tabular}

Table 1. gives a clear picture of the Q-factor for the loose coupling gap and Quasi-linear coupling and the comparison between them. The Q-factor in the loose coupling has a lower value at the first mode in comparison with other modes. This could be due to the coupling loss of the gaps between the feed lines and ring resonator as indicated in [19]. Thus, a Quasi-linear coupling is used in order to overcome this issue. As a result, the Q-factor had a much better result which peaked 141.54 at $2.3 \mathrm{GHz}$ with low insertion loss $-10.51 \mathrm{~dB}$ compared to $-35.19 \mathrm{~dB}$ in the loose coupling. The result of the return loss is slightly enhanced using Quasi-linear coupling when compared to the loose coupling gap at different modes. Resulting values from the table give an idea about the formation of enhancement that reduced the reflected losses using Quasi-linear coupling.

\section{B. Result of loaded overlay sample}

Simulation result for the effect of the thickness of overlay sample on the resonant frequency is presented in Fig.4. Thickness of the overlay samples, in the range of $0.787 \mathrm{~mm}$ to $7.78 \mathrm{~mm}$, is used to be tested in the microstrip ring resonator. From the results it can be observed that a small thickness causes a smaller shift from the resonator's inherent frequency. This happens because of the increasing of the perturbation when the overlay sample size is increased and more fringing fields are focused into the overlay sample as discussed in literature [19], [20]. In Fig.5., results are presented for an amount of attaining resonance shifts for each corresponding thickness of overlay sample. We observed that the low size of the overlay sample shows less significant resonance shift than the empty resonator. The thickness in $0.787-3.148 \mathrm{~mm}$ ranges shows less than $22 \%$ resonance-shift, compared to the height thickness. This is due to the fact that the maximum fields are perturbed by an overlay meat sample. The height of the thickness will lead to the maximum perturbation which causes a higher shift in the resonance frequency, whereas, the low thickness of the overlay meat sample causes a small shift in resonance frequency. 


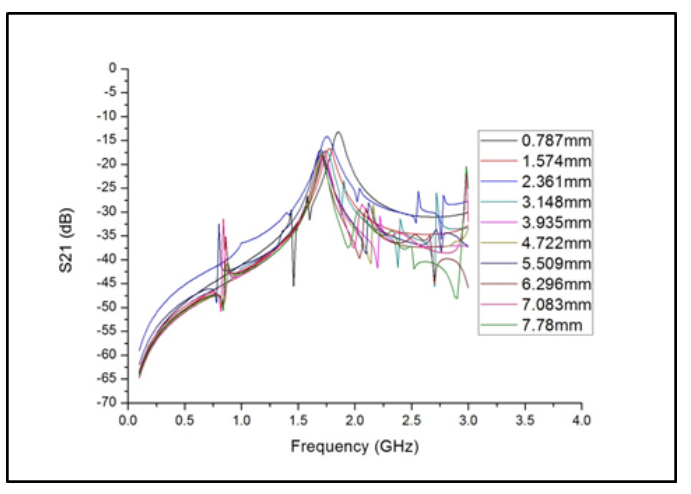

a)

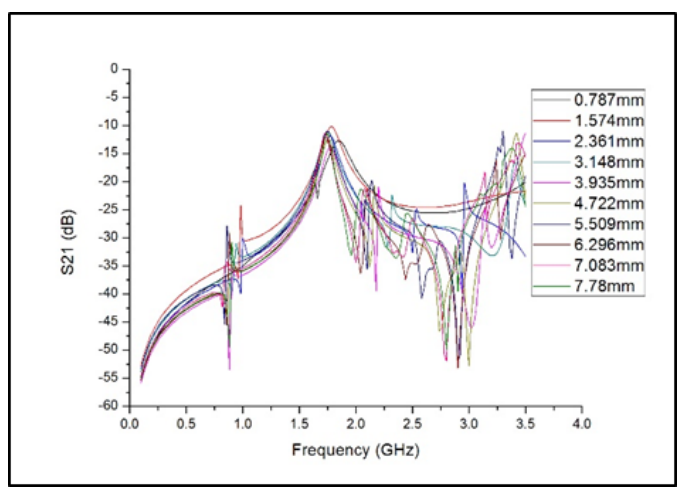

b)

Fig.4. Resonance frequency as a function of multiple overlay thickness: a) using the loose coupling scheme, b) using Quasilinear scheme.

Furthermore, the simulation result is analyzed for the sample overlay ring resonator over the range from $0.787 \mathrm{~mm}$ to $7.78 \mathrm{~mm}$. This range is chosen, as the ratio between the thickness of the overlay sample h2 and the Roger 5880 substrate h1 is equal to 1 to 15 as indicated in [6], the size of the meat sample will not affect significantly the effective permittivity. The resonant frequency and insertion loss are presented as a function of overlay thickness, respectively, which are demonstrated in Fig.4. and Fig.5. Considering Fig.4., it illustrates the thickness of the overlay sample with resonance frequency and when increasing the overlay sample thickness, the shift in resonance frequency is increased. The reason behind this is that the overlay sample size increases, the field perturbation is also increased due to the more fringing fields which are getting concentrated into overlay sample. As a result of increasing fringing field capacitance, the resonance frequency is decreased. Even though there is a shift in resonance frequency, which is significant within a certain thickness, it is limited to lower range only, its effect is not important. Therefore, further increase in sample thickness will not affect the resonance frequency. This is due to the fact that all of the electric field is confined into the overlay sample and substrates which interpret the effective permittivity that increases with the height of the overlay until it reaches the asymptotic value as discussed in literature [20]. Correspondingly, this happens to the insertion loss magnitude as indicated in Fig.6.

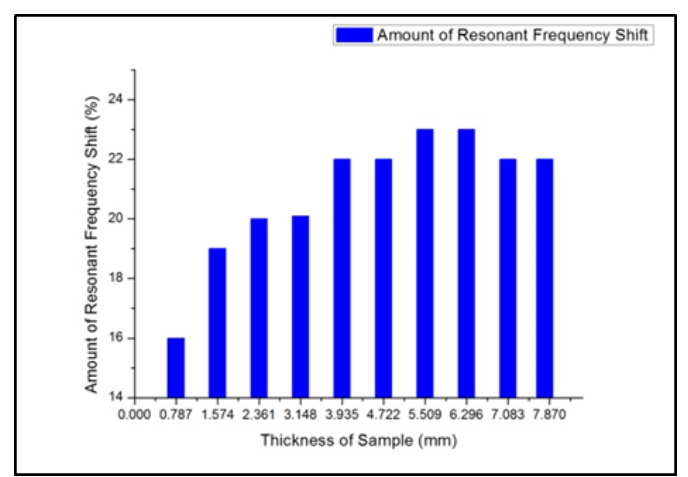

a)

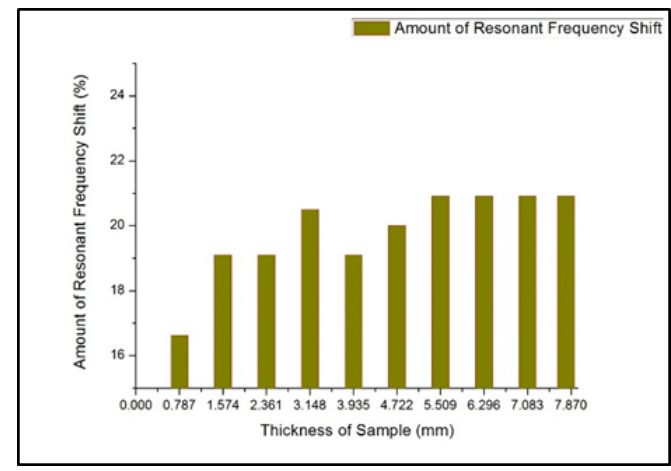

b)

Fig.5. The amount of shift frequency over the range of overlay thickness: a) using loose coupling, b) using Quasi-linear coupling.

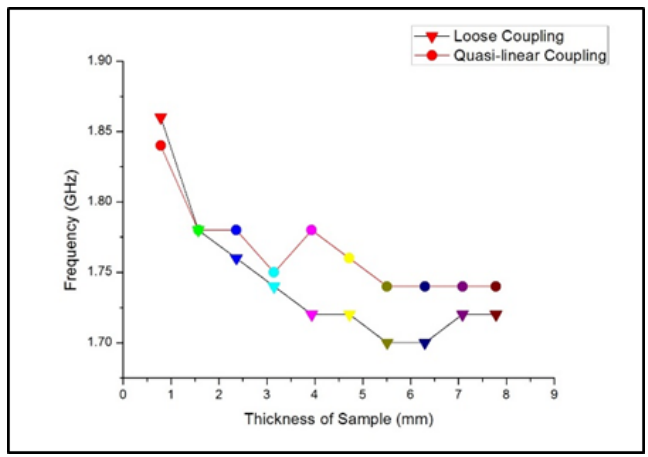

a)

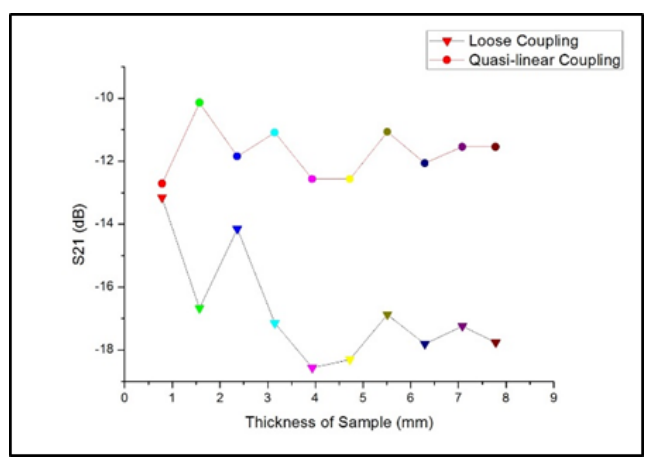

b)

Fig.6. The effect of overlay thickness on: a) resonance frequency, b) Insertion loss S21 in dB. 


\section{Effect of the coupling gap}

The gap has a significant effect on determination of the dielectric properties. The fields of microstrip ring resonator are noticeably disturbed with a small coupling gap while in larger coupling gap size it is less disturbed with high losses in the coupling gap region. Thus, the variations of the coupling gaps were evaluated using a gap size of 0.15 to $0.35 \mathrm{~mm}$ as indicated in Fig.7. It can be observed that the performance of the resonator depends on the size of the coupling gap.

The comparison results before and after optimization for loose coupling scheme are demonstrated in Fig.8. It can be observed that the Q-factor in the SSRR sensor has low value (33.98) at $2.26 \mathrm{GHz}$ operating frequency compared to the other harmonic resonant frequencies before the optimization was made. After the optimization is done for the coupling gap between the ring and the feed lines, a maximum field distribution is produced at $2.26 \mathrm{GHz}$ operating frequency and provides minimum insertion loss. It demonstrates that the SSRR sensor has achieved a high Q-factor around 407.34 at the same operating frequency with smaller insertion loss. A comparison before and after optimization of the loose coupling scheme is indicated in Table 3.

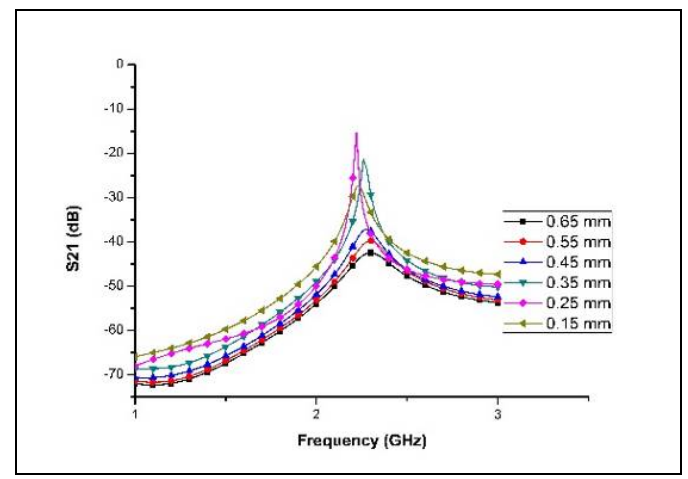

Fig.7. The effect of the coupling gap.

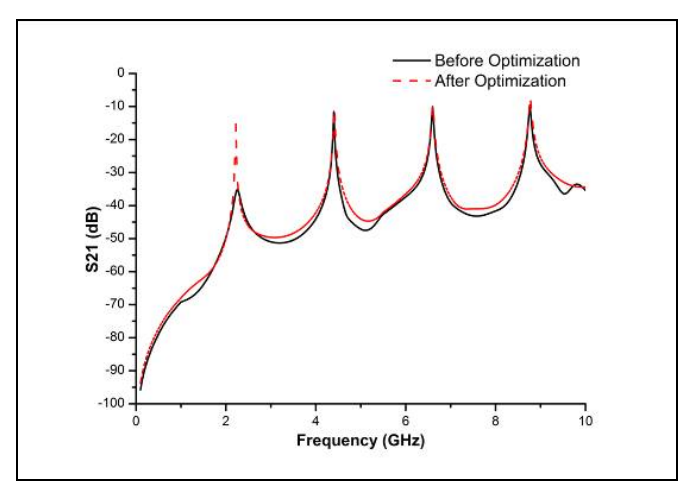

Fig.8. Simulation result of loose coupling before and after optimization.

Fig.9. illustrates the simulation result for quasi-linear coupling scheme before and after the optimization. It can be observed that the Q-factor in quasi-linear coupling scheme has a high value of 278.78 at $2.30 \mathrm{GHz}$ operating frequency after the optimization was made. Table 4 . shows comparison of simulation results before and after optimization for quasilinear coupling scheme.
Table 3. Comparison results before and after optimization for loose coupling scheme.

\begin{tabular}{|c|c|c|c|c|}
\hline \multirow{2}{*}{$\begin{array}{c}\text { Mode } \\
\text { (n) }\end{array}$} & \multicolumn{4}{|c|}{ Loose Coupling After Optimization } \\
\hline & $\begin{array}{c}\text { Frequency } \\
\text { (GHz) }\end{array}$ & $\begin{array}{c}\text { Q- } \\
\text { Factor }\end{array}$ & $\begin{array}{l}\text { S11 } \\
(\mathrm{dB})\end{array}$ & $\begin{array}{c}\text { S21 } \\
\text { (dB) }\end{array}$ \\
\hline 1 & 2.22 & 407.34 & -1.53 & -15.32 \\
\hline 2 & 4.42 & 243.53 & -3.16 & -12.03 \\
\hline 3 & 6.60 & 368.72 & -6.48 & -8.99 \\
\hline 4 & 8.76 & 302.07 & -5.30 & -8.64 \\
\hline
\end{tabular}

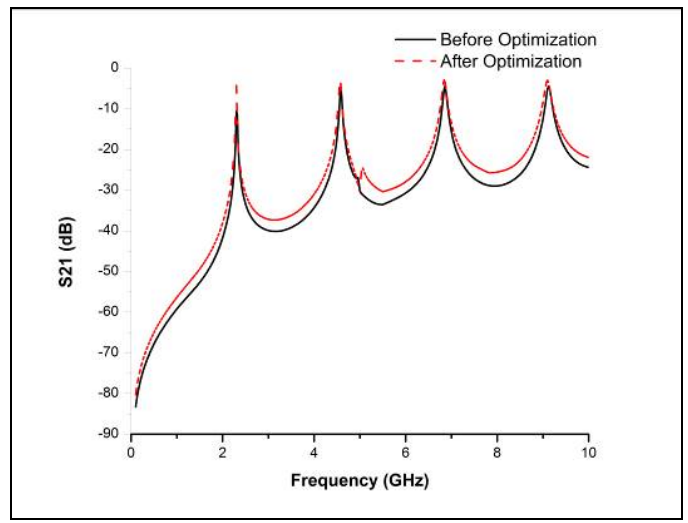

Fig.9. Simulation result of quasi-linear coupling before and after optimization.

Table 3. Comparison results before and after optimization for loose coupling scheme.

\begin{tabular}{|c|c|c|c|c|}
\hline \multirow{2}{*}{$\begin{array}{c}\text { Mode } \\
\text { (n) }\end{array}$} & \multicolumn{4}{|c|}{ Quasi-Linear Coupling After Optimization } \\
\hline & $\begin{array}{c}\text { Frequency } \\
\text { (GHz) }\end{array}$ & $\begin{array}{c}\text { Q- } \\
\text { Factor }\end{array}$ & $\begin{array}{l}\text { S11 } \\
\text { (dB) }\end{array}$ & $\begin{array}{l}\text { S21 } \\
(d B)\end{array}$ \\
\hline 1 & 2.30 & 278.78 & -6.08 & -4.26 \\
\hline 2 & 4.57 & 183.17 & -10.99 & -2.83 \\
\hline 3 & 6.85 & 151.72 & -11.48 & -2.93 \\
\hline 4 & 9.11 & 142.46 & -10.95 & -3.13 \\
\hline
\end{tabular}

\section{Calculation of Real Part Permittivity}

Various dielectric samples from 1 to 10 were loaded and simulated in the proposed SSRR sensor in order to visualize the design of sensing applications. A cube overlay sample with dimension of $25 \times 17 \times 1 \mathrm{~mm}$ ( $\mathrm{L} \mathrm{x} \mathrm{W} \mathrm{x} \mathrm{h)} \mathrm{is} \mathrm{used} \mathrm{in}$ order to model fresh meat along with er 56.5 dielectric constant [21]. Fig.10. demonstrates the resonance frequency which is shifted down accordingly when the value of the dielectric constant of the sample is increased. This is due to the high capacitance when introducing the dielectric material. By referring to (4), increasing the capacitance will produce a low resonance frequency. The shifts of resonance frequency are considered as data which are related to the permittivity of the MUT $(\varepsilon)$. Thus, an expression for the relationship between the permittivity, $\varepsilon$ and frequency, $\mathrm{f}$ of the MUT is required. This expression can be modeled by using curve fitting method based on the data presented in Fig.10. and the polynomial presented in the figure is obtained as follows:

$$
\varepsilon_{r}=85.06 f^{2}-425.69 f+525.27
$$




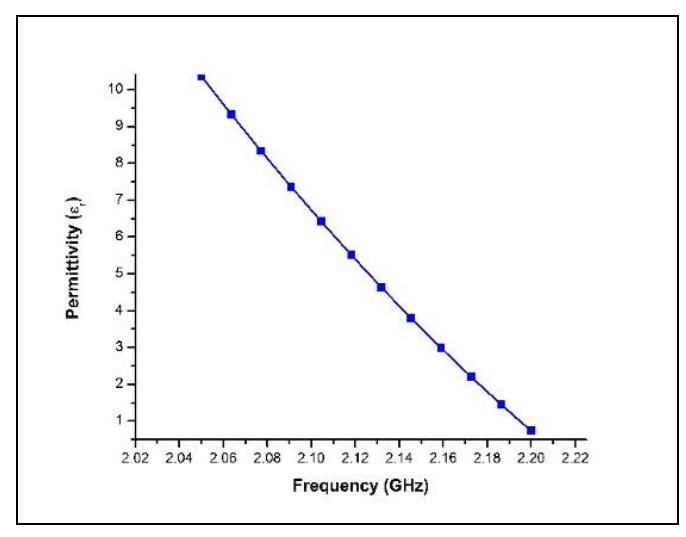

Fig.10. MUT permittivity as a function of resonance frequency for the proposed SSRR sensor.

\section{E. Fabrication and Measurement Results}

The SSRR sensor is fabricated on the Roger RT/Duroid 5880 substrate with 2.2 dielectric constant and $0.787 \mathrm{~mm}$ thickness. Fig.11. shows the fabricated SSRR loose coupling sensor without and with overlay meat sample. It obviously illustrates that the SSRR sensor has narrower band and sharper dip which reveals its high Q nature of around 407.34 compared to other structures. Furthermore, the results demonstrate some small deviations between the simulation and measurement. The measured resonance frequencies are slightly shifted from the simulation and the insertion loss magnitude is lower than the simulated one. This is because of mismatch between the feed-lines and SMA connectors and also the tolerance of fabrications which limits simulation accuracy. The simulation results can be compared with experiment results for both schemes: loose coupling and quasi-linear coupling. The first conclusion of loose coupling is that results are in good agreement, implying that experimental results are valid within the range of simulation results as indicated in Fig.12.a). This implies that the quasi-linear coupling simulation results will be in good agreement with the measurement results.

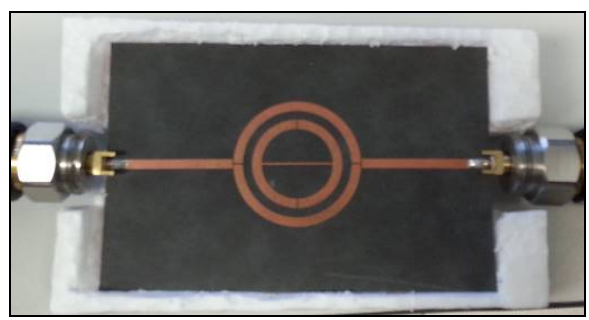

a)

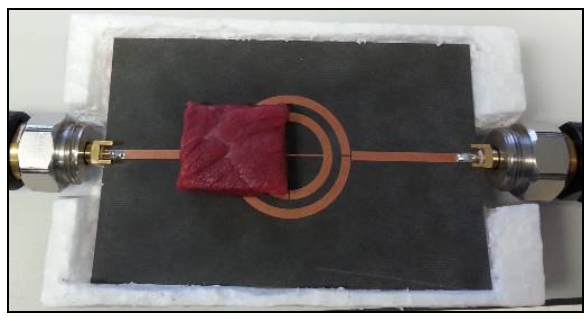

b)

Fig.11. Fabricated SSRR sensor a) without meat sample b) with overlay meat sample.
For simulation results validation, measurements were taken into account with fabricated SSRR sensor. The response behavior of resonance frequency is observed with and without overlay meat sample as indicated in Fig.12.b).

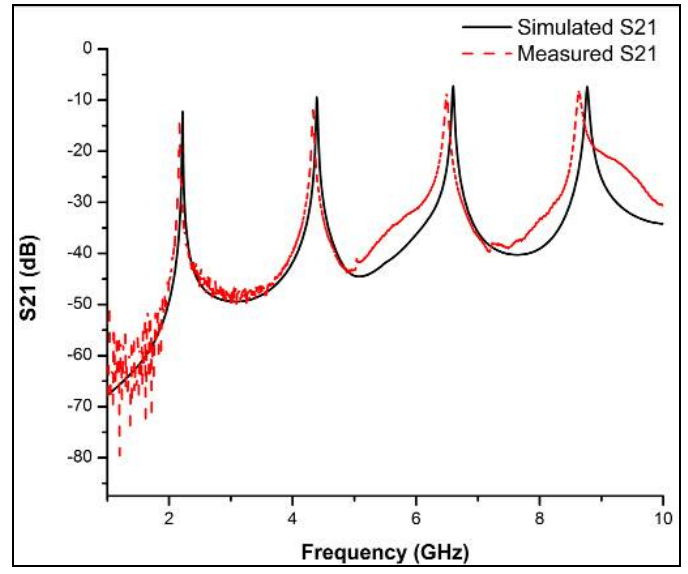

a)

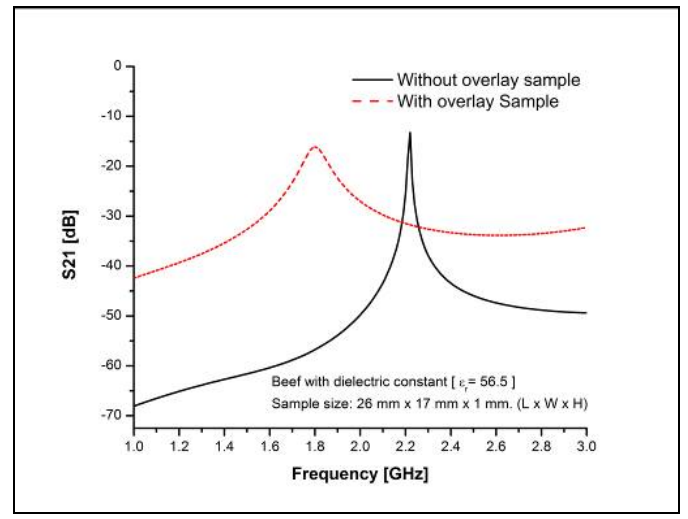

b)

Fig.12. Measured S21 parameter a) Comparison between simulation and measurement results b) Behavior response of resonance frequency with/out overlay fresh meat sample.

\section{CONCLUSION}

In this paper, we have proposed SSRR as a viable candidate for performance enhancement of microwave sensors. We have designed, simulated and tested overlay sample using HFSS simulator. The thickness of the overlay sample was varied and the result was obtained and recorded. The main contribution of this paper is to have a high Qfactor with small insertion loss at the designed operating frequency. This feature is expected to be particularly useful for characterizing materials of solids and bio-sensing applications. The SSRR is used because it is cost effective, simple, and easy to fabricate, easy to simulate, easy to enhance the accuracy of the sensor with respect to material characterization compared to other techniques.

\section{ACKNOWLEDGMENT}

This work was supported by UTeM Zamalah Scheme and funded by UTeM and the Malaysian Government (MOHE) for research grant RAGS/1/2014/TK03/UTEM/FTKB00078. 


\section{REFERENCES}

[1] Rammah, A.A., Zakaria, Z., Ruslan, E., Isa, A.A.M. (2015). Comparative study of materials characterization using microwave resonators. Australian Journal of Basic and Applied Sciences, 9, 76-85.

[2] Jilani, M.T., Zaka, M., Khan, A.M., Khan, M.T., Ali, S.M. (2012). A brief review of measuring techniques for characterization of dielectric materials. ITEE Journal, 1 (1).

[3] Yanbing Ma, Huaiwu Zhang, Yuanxun Li, Yicheng Wang, Weien Lai. (2013). Terahertz sensing application by using fractal geometries of split-ring resonators. Progress In Electromagnetics Research, 138, 407-419.

[4] Alahnomi, R.A., Zakaria, Z., Ruslan, E., Isa, A.A.M. (2015). Optimization analysis of microwave ring resonator for bio-sensing application. International Journal of Applied Engineering Research, 10 (7), 18395-18406.

[5] Taha Jilani, M., Wong, P.W., Zakariya, M.A., Lee, Y.C. (2014). Dielectric characterization of meat using enhanced coupled ring-resonator. In IEEE Asia-Pacific Conference on Applied Electromagnetics, 8-10 December 2014. IEEE, Vol. 1, 191-194.

[6] Jilani, M.T., Wen, W.P., Zakariya, M.A., Cheong, L.Y. Dielectric method for determination of fat content at $1 \mathrm{GHz}$ frequency. In 5th International Conference on Intelligent and Advanced Systems (ICIAS), 3-5 June 2014. IEEE, 1-4.

[7] Saeed, K., Pollard, R.D., Hunter, I.C. (2008). Substrate integrated waveguide cavity resonators for complex permittivity characterization of materials. IEEE Transactions on Microwave Theory and Techniques, 56 (10), 2340-2347.

[8] Aygun, K., Hu, G. (2008). Characterization of halogen-free package materials using cavity resonators. In IEEE-EPEP Electrical Performance of Electronic Packaging, 27-29 October 2008. IEEE, 147-150.

[9] Jacob, M.V., Thomas, S., Sebastian, M., Mazierska, J., Honkama, J., Jantunen, H. (2008). Low temperature microwave characterisation of greentapes using Split Post Dielectric Resonator. In Asia-Pacific Microwave Conference (APMC 2008), 16-20 December 2008. IEEE, Vol. 2, 1-4.

[10] Schaub, D.E., Oliver, D.R. (2011). A circular patch resonator for the measurement of microwave permittivity of nematic liquid crystal. IEEE Transactions on Microwave Theory and Techniques, 59 (7), 1855-1862.

[11] Rashidian, A., Aligodarz, M., Klymyshyn, D. (2012). Dielectric characterization of materials using a modified microstrip ring resonator technique. IEEE Transactions on Dielectrics and Electrical Insulation, 19 (4), 1392-1399.
[12] Zelenchuk, D.E., Fusco, V., Goussetis, G., Mendez, A., Linton, D. (2012). Millimeter-wave printed circuit board characterization using substrate integrated waveguide resonators. IEEE Transactions on Microwave Theory and Techniques, 60 (10), 33003308.

[13] Kilic, E., Siart, U., Wiedenmann, O., Faz, U., Ramakrishnan, R., Saal, P., Eibert, T.F. (2013). Cavity resonator measurement of dielectric materials accounting for wall losses and a filling hole. IEEE Transactions on Instrumentation and Measurement, 62 (2), 401-407.

[14] Córdoba-Erazo, M.F., Weller, T.M. (2012). Liquids characterization using a dielectric resonator-based microwave probe. In 42nd European Microwave Conference (EuMC), 29 October - 1 November 2012. IEEE, 655-658.

[15] Zhu, X.-C., Hong, W., Wu, K., Tang, H.-J., Hao, Z.C., Zhou, H.-X. (2013). Characterization of substrate material using complementary split ring resonators at terahertz frequencies. In IEEE International Wireless Symposium (IWC), 14-18 April 2013. IEEE, 1-4.

[16] Aouabdia, N., Belhadj-Tahar, N.E., Alquie, G. (2014). Rectangular patch resonator sensors for characterization of biological materials. In 11th International Multi-Conference on Systems, Signals \& Devices (SSD), 11-14 February 2014. IEEE, 1-4.

[17] Zhou, J., Jia, P., Zhang, Y., He, X. (2013). High sensitive biosensor based on aSRR and highimpedance microstrip line. In International Conference on Measurement, Information and Control (ICMIC), 16-18 August 2013. IEEE, 234-237.

[18] Ansari, M.A.H., Jha, A.K., Akhtar, M.J. (2015). Permittivity measurement of common solvents using the CSRR based sensor. In IEEE International Symposium on Antennas and Propagation \& USNC/URSI National Radio Science Meeting, 19-24 July 2015. IEEE, 1199-1200.

[19] Chang, K., Hsieh, L.H. (2004). Microwave Ring Circuits and Related Structures, 2nd Ed. John Wiley \& Sons.

[20] Jilani, M.T., Wen, W.P., Cheong, L.Y., Rehman, M.Z.U., Khan, M.T. (2016). Determination of sizeindependent effective permittivity of an overlay material using microstrip ring. Microwave and Optical Technology Letters, 58 (1), 4-9.

[21] Clerjon, S., Damez, J. (2009). Microwave sensing for an objective evaluation of meat ageing. Journal of Food Engineering, 94 (3-4), 379-389.

Received August 14, 2015. Accepted January 28, 2016. 